\title{
Magnetic and acoustic properties of $\mathrm{CoCr}_{2} \mathrm{~S}_{4}$
}

\author{
V. Felea ${ }^{1}$, P.T. Cong ${ }^{2}$, L. Prodan ${ }^{1}$, Y. Gritsenko ${ }^{2,3}$, J. Wosnitza $^{2,3}$, \\ S. Zherlitsyn ${ }^{2}$, and V. Tsurkan ${ }^{1,4}$ \\ ${ }^{1}$ Institute for Applied Physics, Academy of Science of Moldova, Chisinau MD-2028, Republic of Moldova \\ ${ }^{2}$ Hochfeld-Magnetlabor Dresden (HLD-EMFL), Helmholtz-Zentrum Dresden-Rossendorf, Dresden D-01314, Germany \\ E-mail: s.zherlitsyn@hzdr.de \\ ${ }^{3}$ Institut für Festkörperphysik, Technische Universität Dresden, Dresden D-01062, Germany \\ ${ }^{4}$ Experimental Physics 5, Center for Electronic Correlations and Magnetism, Institute of Physics, \\ Universität Augsburg, Augsburg D-86159, Germany
}

Received May 25, 2017, published online September 25, 2017

\begin{abstract}
We report results of magnetic and ultrasound studies of the sulfide spinel $\mathrm{CoCr}_{2} \mathrm{~S}_{4}$, for which the multiferroicity has recently been suggested. Clear anomalies in the magnetic and acoustic properties have been observed at $T_{N}=222 \mathrm{~K}$ and in applied magnetic fields evidencing the important role of magnetoelastic interactions in this material. In contrast, no anomalies have been detected at $T_{C}=28 \mathrm{~K}$, where a spontaneous electric polarization and isostructural distortions have been reported. We have extracted the $H-T$ phase diagram of $\mathrm{CoCr}_{2} \mathrm{~S}_{4}$ from our experiments for magnetic fields applied along the $<111>$ direction. We discuss our observations in relation to our earlier results obtained for the oxide multiferroic spinel $\mathrm{CoCr}_{2} \mathrm{O}_{4}$.
\end{abstract}

PACS: 72.55.+s Magnetoacoustic effects;

62.65.+k Acoustical properties of solids;

75.50.Ee Antiferromagnetics.

Keywords: sulfide spinel $\mathrm{CoCr}_{2} \mathrm{~S}_{4}$, magnetic and acoustic properties, electric polarization.

Frustrated magnetic systems are of great interest exhibiting exotic magnetic states and unusual cooperative phenomena. In three dimensions, a typical example of such frustrated systems is a pyrochlore-lattice material with the magnetic ions located on the vertices of corner-sharing tetrahedra and having competing magnetic interactions. Spinels with $\mathrm{Cr}^{3+}$ ions, $\mathrm{ACr}_{2} \mathrm{X}_{4}$ ( $\mathrm{A}=\mathrm{Mn}, \mathrm{Cd}, \mathrm{Co}, \mathrm{Zn}$ and $\mathrm{X}=\mathrm{O}, \mathrm{S}$, Se) are ideal candidates for studying various magnetic frustration effects. For these spinels a strong spin-phonon coupling frequently leads to clearly visible spin-lattice effects [1-10]. The oxide spinels are geometrically frustrated with a direct antiferromagnetic (AFM) exchange of the order of a few hundred Kelvin, whereas the sulfide and selenide spinels are bond frustrated due to a competition between the direct AFM exchange and the $90^{\circ}$ ferromagnetic (FM) exchange interactions, still having an AFM ground state [11].

Remarkable example of the oxide spinels is $\mathrm{CoCr}_{2} \mathrm{O}_{4}$. This material exhibits a number of unique magnetic states including commensurate and incommensurate spin configurations, a high-field phase with a disordered transverse component of the magnetization above $43 \mathrm{~T}$, and a huge metastable region in the $H-T$ phase diagram [3]. Furthermore, a spontaneous dielectric polarization appears in the incom- mensurate-spiral state below $27 \mathrm{~K}$ [12]. This multiferroicity in $\mathrm{CoCr}_{2} \mathrm{O}_{4}$ was assigned to the inverse Dzyaloshinskii-Moriya interaction based on the fact that the observed modulations of the spins and the lattice have the same wave vector [13].

A closely related material is the sulfide counterpart $\mathrm{CoCr}_{2} \mathrm{~S}_{4}$, which has a cubic structure $(\mathrm{Fd} \overline{3} \mathrm{~m})$ with $\mathrm{Cr}^{3+}$ $\left(3 d^{3}, S=3 / 2\right)$ ions situated at octahedral positions and $\mathrm{Co}^{2+}\left(3 d^{7}, S=3 / 2\right)$ occupying tetrahedral sites. $\mathrm{CoCr}_{2} \mathrm{~S}_{4}$ undergoes a ferrimagnetic transition at $T_{N}=222 \mathrm{~K}$ in a state with the $\mathrm{Co}^{2+}$ and $\mathrm{Cr}^{3+}$ sublattices aligned antiparallel to each other since the $\mathrm{Co}-\mathrm{Cr}$ interactions are stronger than the Co-Co or Cr-Cr ones [14]. The magnetic properties of $\mathrm{CoCr}_{2} \mathrm{~S}_{4}$ have been studied in Refs. 14-16. Hydrostatic pressure results in a linear increase of the ordering temperature by $0.5 \mathrm{~K} / \mathrm{kbar}$ due to the increase of the dominant nearestneighbor Co-Cr superexchange interaction [17], hinting at the important role of the spin-strain coupling in this material. Recently, in polycrystalline $\mathrm{CoCr}_{2} \mathrm{~S}_{4}$ polar order below $28 \mathrm{~K}$ has been reported [18] suggesting multiferroicity of this material with a spontaneous electric polarization 60 times larger than that in the oxide spinel $\mathrm{CoCr}_{2} \mathrm{O}_{4}$. Moreover, a strong magnetoelastic coupling at $T_{N}$ and isostructural 
distortions at $28 \mathrm{~K}$, enhanced by external magnetic fields, have been suggested based on synchrotron powderdiffraction studies [18]. The occurrence of polar order involves the expansion of the Co tetrahedra and contraction of the Cr octahedra in the spinel structure. The appearance of a spiral spin order below $28 \mathrm{~K}$ has been suggested as well [18].

In this work we report results of magnetization and ultrasound experiments on $\mathrm{CoCr}_{2} \mathrm{~S}_{4}$ single crystals. We compare our observations with those of the closely related multiferroic spinel $\mathrm{CoCr}_{2} \mathrm{O}_{4}$ [3]. We further investigated the role of the magnetoelastic couplings on the physics of $\mathrm{CoCr}_{2} \mathrm{~S}_{4}$. The ultrasound technique is a sensitive tool for detecting magnetoelastic couplings and structural phase transitions [19]. Spin-lattice effects originate mainly from the exchange-striction mechanism caused by the renormalization of inter-atomic magnetic interactions due to spin-phonon coupling. In this case the sound velocity and sound attenuation changes are related to the magnetization and non-uniform magnetic susceptibilities with the renormalization being proportional to the spin-phonon coupling constants [2,6,20,21].

High-quality $\mathrm{CoCr}_{2} \mathrm{~S}_{4}$ single crystals were grown by chemical transport reactions. The phase purity of the sample was checked by $\mathrm{x}$-ray analysis. The shape of the single crystal allowed us to propagate acoustic waves along the $<111>$ direction. Two opposite (111)-crystal surfaces were polished for the ultrasound experiments. The sample thickness along the $<111>$ direction was $1.28 \mathrm{~mm}$. The elastic properties were studied by measurements of the velocity and attenuation of longitudinal waves with the wave vector $\mathbf{k}$ and polarization $\mathbf{u}$ parallel to the $<111>$ axis, which for a cubic crystal correspond to the elastic constant $c_{L}=\left(c_{11}+2 c_{12}+4 c_{44}\right) / 3$. A phase-sensitive detection technique based on a pulse-echo method [21] was used. The sound velocity $v(\mathbf{k}, \mathbf{u})$ is related to the elastic modulus via $c_{i j}=\rho[v(\mathbf{k}, \mathbf{u})]^{2}$, where $\rho$ is the mass density of the crystal and $\Delta v / v=\left[v(T, H)-v_{0}\right] / v_{0}$, with the sound velocity $v_{0}$ at the initial value of the external parameters $T$ and $H$. Note, that $c_{L}$ is a pure acoustic mode which involves all three elastic constants of the cubic crystal. Wide-band polyvinylidene fluoride (PVDF) films were used to generate and detect longitudinal acoustic waves. The magnetic field was applied along the $<111>$ direction. The measurements in static magnetic fields up to $6 \mathrm{~T}$ were performed for temperatures between 1.5 and $300 \mathrm{~K}$. Temperatures down to $1.5 \mathrm{~K}$ were reached by use of ${ }^{4} \mathrm{He}$ cryostats placed inside of a $20 \mathrm{~T}$ superconducting magnet or a $65 \mathrm{~T}$ pulsed magnet with the total pulse duration of $\sim 150 \mathrm{~ms}[22,23]$. $\mathrm{RuO}_{2}$ and PT100 thermometers were thermally coupled to the sample. The magnetization was measured by use of a SQUID magnetometer (Quantum Design MPMS-5) in static fields up to $5 \mathrm{~T}$.

The inset of Fig. 1 shows the temperature dependence of the magnetization obtained below $400 \mathrm{~K}$ in a magnetic field of $50 \mathrm{G}$ under field-cooled (FC) and zero-field-cooled (ZFC) conditions. A finite spontaneous magnetization appears below $T_{N}=222 \mathrm{~K}$ with a different response for the

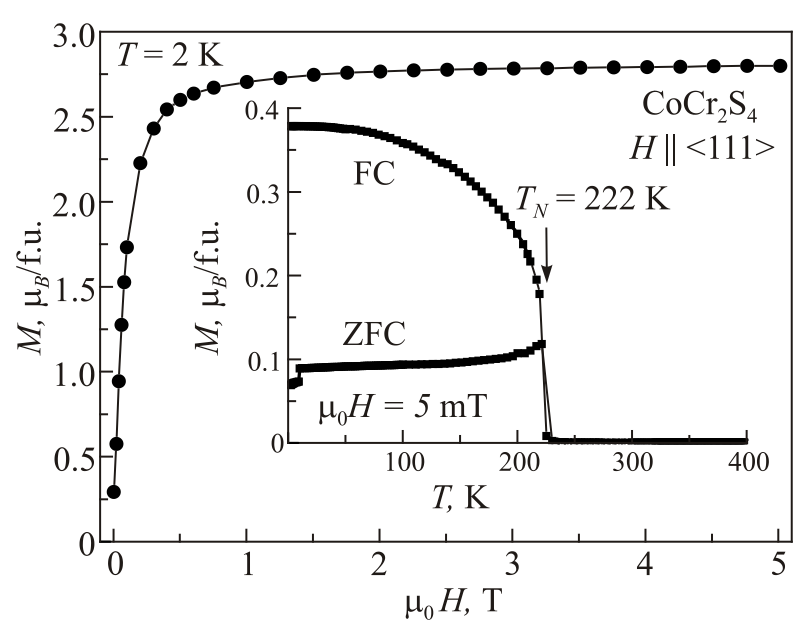

Fig. 1. Magnetization of $\mathrm{CoCr}_{2} \mathrm{~S}_{4}$ vs magnetic field measured at $2 \mathrm{~K}$ for the magnetic field applied along the $<111>$ direction. The inset shows the temperature dependence of the magnetization measured in a magnetic field of $5 \mathrm{mT}$ applied along the $<111>$ direction. Results obtained for the FC and ZFC conditions are shown. The ferrimagnetic ordering at $T_{N}=222 \mathrm{~K}$ is marked by an arrow.

FC and ZFC conditions. Such magnetization anomalies are typical for the ferrimagnetic ordering reported in $\mathrm{CoCr}_{2} \mathrm{~S}_{4}$ at about the same temperature earlier $[14,15]$. Similar magnetization results have been previously obtained for a polycristalline sample [18] as well as for a single crystal [16]. No magnetization anomalies have been observed down to $2 \mathrm{~K}$ neither in our investigation nor in previous works $[15,16]$, although clear anomalies have been detected in $\mathrm{CoCr}_{2} \mathrm{O}_{4}$ at the transition into the incommensurate spiral-spin state at $T_{S} \approx 27 \mathrm{~K}$ with a spontaneous dielectric polarization [3]. The main panel of Fig. 1 shows the field-induced magnetization obtained at $2 \mathrm{~K}$. The magnetization saturates above $\approx 1 \mathrm{~T}$ at the level of $2.7 \mu_{B}$ per formula unit. This is in a good agreement with the calculated saturation magnetization of 2.4-2.7 $\mu_{B}$ per formula unit with 3.3-3.6 $\mu_{B}$ of $\mathrm{Co}^{2+}$ opposing about $6 \mu_{B}$ on the $\mathrm{Cr}^{3+}$ ions [15].

Figure 2 shows the temperature dependence of the sound velocity for the acoustic mode $c_{L}$ in $\mathrm{CoCr}_{2} \mathrm{~S}_{4}$. Due to usual anharmonic contributions, there is a characteristic increase in the sound velocity by lowering the temperature. The sound velocity exhibits a slope change (kink) at $T_{N}$ with an increased stiffness appearing in the ordered state. These features are clear manifestations of the magnetoelastic interactions in $\mathrm{CoCr}_{2} \mathrm{~S}_{4}$. Moderate magnetic fields shift $T_{N}$ to higher temperatures broadening the corresponding soundvelocity anomaly (inset of Fig. 2). No acoustic anomaly has been detected at $28 \mathrm{~K}$ where a change of magnetic structure, polar order, and an isostructural distortion have been suggested [18]. This is puzzling, since the acoustic properties are very sensitive to structural and magnetic transitions as shown in various spinels [2-6]. The specific heat results obtained in polycrystalline $\mathrm{CoCr}_{2} \mathrm{~S}_{4}$ do not show any anomaly at $28 \mathrm{~K}$ as well [24]. Note, that a pronounced anomaly in the 


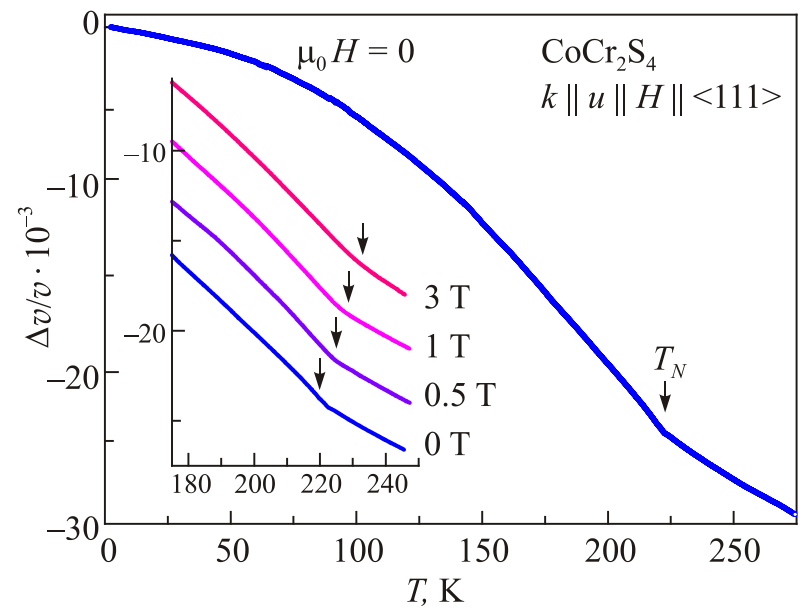

Fig. 2. (Color online) Temperature dependence of the soundvelocity changes, $\Delta v / v$, in $\mathrm{CoCr}_{2} \mathrm{~S}_{4}$ for the longitudinal $c_{L}$ mode measured in zero magnetic field. The inset shows the data near $T_{N}$ (marked by arrows) in magnetic fields applied along $<111>$. The data for different magnetic fields are shifted along the $y$ axis for clarity. The ultrasound frequency was $60 \mathrm{MHz}$.

sound velocity has been observed in $\mathrm{CoCr}_{2} \mathrm{O}_{4}$ at the transition into the incommensurate spiral-spin state at $T_{S} \approx 27 \mathrm{~K}$, where a spontaneous dielectric polarization appears [3]. Moreover, a strong softening of $c_{L}$ of about $4 \%$ has been observed as a precursor of the multiferroic state in $\mathrm{CoCr}_{2} \mathrm{O}_{4}$. One may speculate whether another acoustic mode might be stronger coupled to the spin subsystem of $\mathrm{CoCr}_{2} \mathrm{~S}_{4}$ than $c_{L}$.

The field dependence of the acoustic properties at $4 \mathrm{~K}$ is shown in Fig. 3. The sound velocity exhibits a sharp, jump-like increase at $\sim 0.5 \mathrm{~T}$ followed by a shallow minimum at about $1.2 \mathrm{~T}$. A negligibly small hysteresis has been observed near $0.5 \mathrm{~T}$. The sound attenuation increases in the same field range, passes through a sharp maximum at 0.75 $\mathrm{T}$, and levels off above $1.5 \mathrm{~T}$. The attenuation maximum coincides with the decrease in $\Delta v / v$ after the jump-like increase. The oscillations in the acoustic properties above 2 $\mathrm{T}$ are apparently the experimental artefacts. At higher fields, a softening of the acoustic mode occurs above $\sim 6 \mathrm{~T}$ such that $c_{L}$ has approximately the same stiffness at 0 and $62 \mathrm{~T}$ (inset of Fig. 3). The observed softening at high magnetic fields is most probably related to the Zeeman splitting of the two low-lying spin-doublets [14] and related level crossovers. These observations evidence that the high field acoustic properties of $\mathrm{CoCr}_{2} \mathrm{~S}_{4}$ are quite different from those of $\mathrm{CoCr}_{2} \mathrm{O}_{4}$ [3].

Figure 4 shows the sound velocity versus magnetic field measured at selected temperatures. The jump in the sound velocity survives in the ordered state at least up to $200 \mathrm{~K}$. Note that the total change of the sound velocity in the magnetic field of about $10^{-3}$ is much less than the velocity change from room temperature down to $1.5 \mathrm{~K}$ in zero magnetic field, $\sim 3 \cdot 10^{-2}$. Interestingly, the data obtained at 20 and $92 \mathrm{~K}$ are quite similar in spite of the suggested polar and spiral spin order below $28 \mathrm{~K}$ in this material [18].

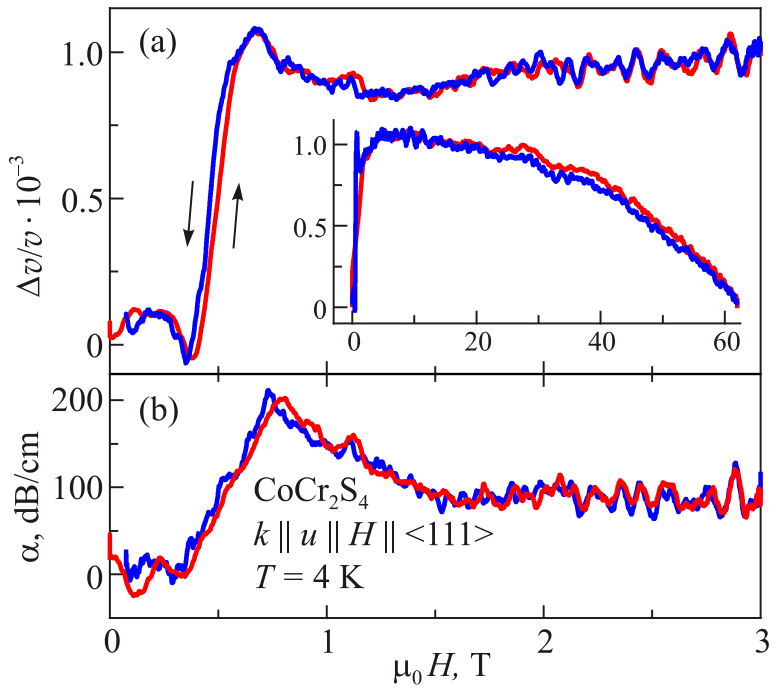

Fig. 3. (Color online) Field dependence of the sound velocity (a) and sound attenuation (b) of the mode $c_{L}$ in $\mathrm{CoCr}_{2} \mathrm{~S}_{4}$ at $4 \mathrm{~K}$. Arrows indicate the field-sweep directions. The magnetic field was applied along <111>, the ultrasound frequency was $53.6 \mathrm{MHz}$. The inset shows the sound-velocity change for the same acoustic mode measured in pulsed magnetic fields up to $62 \mathrm{~T}$ at the same temperature and frequency as in the main figure.

In Fig. 5, we plot the $H-T$ phase diagram with the positions of the anomalies extracted from our ultrasound data. The black squares are from the jumps in the sound velocity (Fig. 4) and the blue triangles reflect the temperature dependent kinks in $\Delta v / v$ in applied magnetic fields (arrows in Fig. 2). The ordering temperature, $T_{N}=(222 \pm 2) \mathrm{K}$ at $H=0$, is extracted from the magnetization (inset of Fig. 1) and the sound velocity (Fig. 2) as well. The magnetic field shifts $T_{N}$ to higher temperatures with $\sim 4 \mathrm{~K} / \mathrm{T}$ (for $\mu_{0} H<1 \mathrm{~T}$ ).

In conclusion, we have studied the magnetic and acoustic properties in the bond-frustrated antiferromagnet $\mathrm{CoCr}_{2} \mathrm{~S}_{4}$

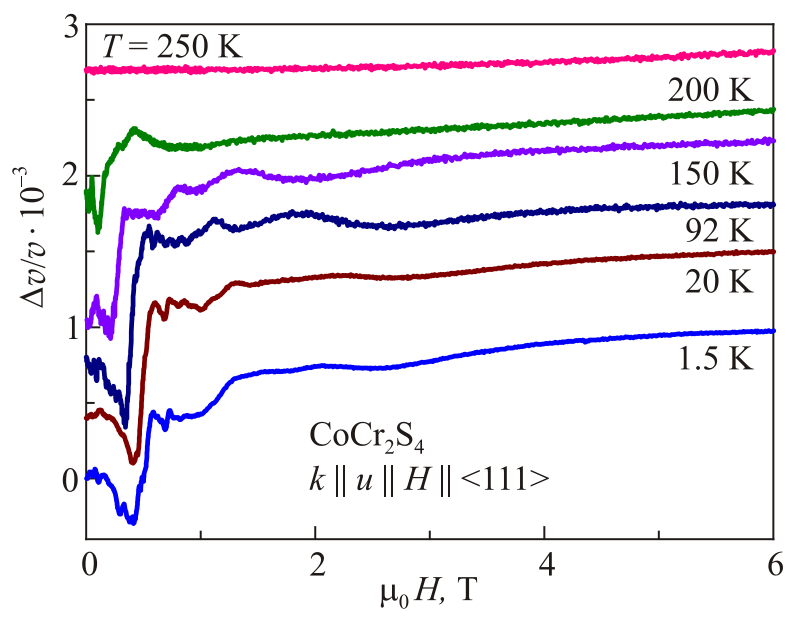

Fig. 4. (Color online) Field dependence of the sound velocity of the mode $c_{L}$ in $\mathrm{CoCr}_{2} \mathrm{~S}_{4}$ measured at selected temperatures. The data for various temperatures are shifted along the $y$ axis for clarity. The ultrasound frequency was $60 \mathrm{MHz}$. The magnetic field was applied along the $<111>$ direction. 


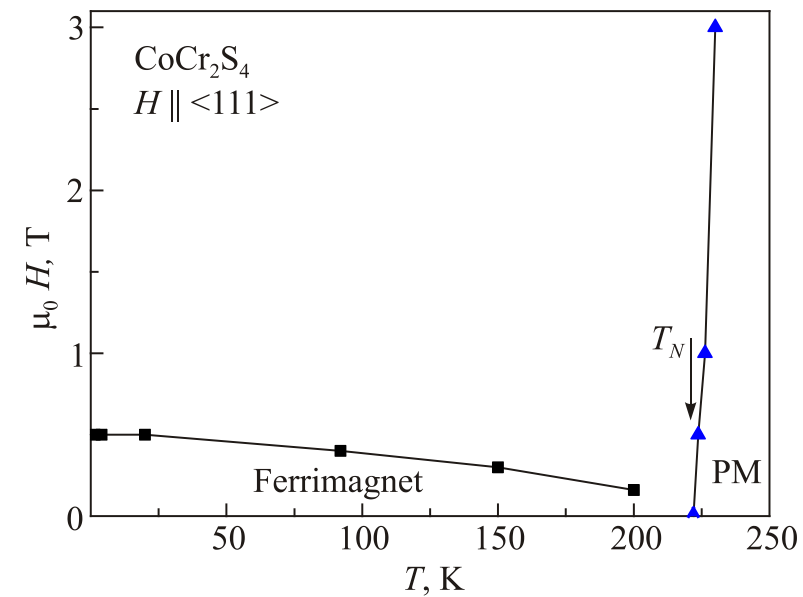

Fig. 5. (Color online) $H-T$ phase diagram of $\mathrm{CoCr}_{2} \mathrm{~S}_{4}$ extracted from ultrasound experiments. The magnetic field is applied along the $<111>$ direction. PM states for the paramagnetic phase.

by means of magnetization and ultrasound experiments. The magnetoelastic coupling plays an important role in this sulfide spinel leading to the renormalization of the acoustic properties at the magnetic phase transition. The magnetization and acoustic anomalies are clearly seen at $T_{N}$ with an additional elastic stiffness appearing in the ordered state. Magnetic fields applied along $<111>$ increase the ordering temperature. A jump-like anomaly has been observed in the sound velocity at $\sim 0.5 \mathrm{~T}$ indicating a field-induced phase transition. A smooth increase in the magnetization at about the same fields might be related to the change of the domain structure. However, the sound-velocity anomaly cannot be explained by change of the domain structure alone. The nature of this transition should be subject of further investigations. The shallow minimum in the sound velocity above $0.7 \mathrm{~T}$ is probably due to the strain interactions with the spin waves [25]. The obtained results are significantly different than those for the closely related multiferroic $\mathrm{CoCr}_{2} \mathrm{O}_{4}$ [3]. All ultrasound anomalies revealed in the sulfide spinel $\mathrm{CoCr}_{2} \mathrm{~S}_{4}$ are much weaker than in the oxide counterpart $\mathrm{CoCr}_{2} \mathrm{O}_{4}$ [3]. No indication for an isostructural phase transition and change of the magnetic structure, as suggested in Ref. 18, has been observed in the magnetic and elastic properties of $\mathrm{CoCr}_{2} \mathrm{~S}_{4}$.

We acknowledge the support of HLD at HZDR, member of the European Magnetic Field Laboratory (EMFL). The research has been supported by the DFG via TRR 80 (Augsburg-Munich) and SFB 1143 (Dresden) and the project for young researches 16.819.02.03F (ASM Moldova).

1. V. Tsurkan, S. Zherlitsyn, L. Prodan, V. Felea, P.T. Cong, Y. Skourski, Z. Wang, J. Deisenhofer, H.-A. Krug von Nidda, J. Wosnitza, and A. Loidl, Sci. Adv. 3, e1601982 (2017).

2. S. Bhattacharjee, S. Zherlitsyn, O. Chiatti, A. Sytcheva, J. Wosnitza, R. Moessner, M.E. Zhitomirsky, P. Lemmens, V. Tsurkan, and A. Loidl, Phys. Rev. B 83, 184421 (2011).
3. V. Tsurkan, S. Zherlitsyn, S. Yasin, V. Felea, Y. Skourski, J. Deisenhofer, H.-A. Krug von Nidda, J. Wosnitza, and A. Loidl, Phys. Rev. Lett. 110, 115502 (2013).

4. V. Tsurkan, S. Zherlitsyn, V. Felea, S. Yasin, Yu. Skourski, J. Deisenhofer, H.-A. Krug von Nidda, P. Lemmens, J. Wosnitza, and A. Loidl, Phys. Rev. Lett. 106, 247202 (2011).

5. V. Felea, S. Yasin, A. Günther, J. Deisenhofer, H.-A. Krug von Nidda, S. Zherlitsyn, V. Tsurkan, P. Lemmens, J. Wosnitza, and A. Loidl, Phys. Rev. B 86, 104420 (2012).

6. S. Zherlitsyn, V. Tsurkan, A.A. Zvyagin, S. Yasin, S. Erfanifam, R. Beyer, M. Naumann, E. Green, J. Wosnitza, and A. Loidl, Phys. Rev. B 91, 060406(R) (2015).

7. A. Miyata, S. Takeyama, and H. Ueda, Phys. Rev. B 87, 214424 (2013).

8. M. Matsuda, K. Ohoyama, S. Yoshii, H. Nojiri, P. Frings, F. Duc, B. Vignolle, G.L.J.A. Rikken, L.-P. Regnault, S-H. Lee, H. Ueda, and Y. Ueda, Phys. Rev. Lett. 104, 047201 (2010).

9. O. Tchernyshyov, R. Moessner, and S.L. Sondhi, Phys. Rev. Lett. 88, 067203 (2002); Phys. Rev. B 66, 064403 (2002).

10. K. Penc, N. Shannon, and H. Shiba, Phys. Rev. Lett. 93, 197203 (2004).

11. T. Rudolf, C. Kant, F. Mayr, J. Hemberger, V. Tsurkan, and A. Loidl, New J. Phys. 9, 76 (2007).

12. Y. Yamasaki, S. Miyasaka, Y. Kaneko, J.-P. He, T. Arima, and Y. Tokura, Phys. Rev. Lett. 96, 207204 (2006).

13. T. Arima, Y. Yamasaki, T. Goto, S. Iguchi, K. Ohgushi, S. Miyasaka, and Y. Tokura, J. Phys. Soc. Jpn. 76, 023602 (2007).

14. P. Gibart, J.-L. Dormann, and Y. Pellerin, Phys. Status Solidi 36, 187 (1969).

15. V. Sagredo, M.C. Moron, and G.E. Delgado, Physica B 384, 82 (2006).

16. K. Ohgushi, Y. Okimoto, T. Ogasawara, S. Miyasaka, and Y. Tokura, J. Phys. Soc. Jpn. 77, 034713 (2008).

17. T. Kanomata, K. Shirakawa, and T. Kaneko, J. Phys. Soc. Jpn. 54, 334 (1985).

18. K. Dey, A. Karmakar, A. Indra, S. Majumdar, U. Rütt, O. Gutowski, M. v. Zimmermann, and S. Giri, Phys. Rev. B 92, 024401 (2015).

19. B. Lüthi, Physical Acoustics in the Solid State, Springer, Berlin (2005).

20. O. Chiatti, A. Sytcheva, J. Wosnitza, S. Zherlitsyn, A.A. Zvyagin, V.S. Zapf, M. Jaime, and A. Paduan-Filho, Phys. Rev. B 78, 094406 (2008).

21. S. Zherlitsyn, S. Yasin, J. Wosnitza, A.A. Zvyagin, A.V. Andreev, and V. Tsurkan, Fiz. Nizk. Temp. 40, 160 (2014) [Low Temp. Phys. 40, 123 (2014)].

22. S. Zherlitsyn, B. Wustmann, T. Herrmannsdörfer, and J. Wosnitza, J. Low Temp. Phys. 170, 447 (2013).

23. S. Zherlitsyn, B. Wustmann, T. Herrmannsdörfer, and J. Wosnitza, IEEE Trans. Appl. Superconduct. 22, 4300603, (2012).

24. A.A. Abdurragimov, M.A. Alyanov, N.G. Guseinov, and A.G . Guseinov, Phys. Status Solidi A 113, K207 (1989).

25. T.A. Kaplan, Phys. Rev. 109, 782 (1958). 